\title{
DECOHERENCE AND ASYMPTOTIC ENTANGLEMENT IN OPEN QUANTUM DYNAMICS
}

\author{
Aurelian Isar \\ National Institute of Physics and Nuclear Engineering \\ Bucharest-Magurele, Romania \\ e-mail: isar@theory.nipne.ro
}

\begin{abstract}
Within the framework of the theory of open systems based on completely positive quantum dynamical semigroups, we determine the degree of quantum decoherence of a harmonic oscillator interacting with a thermal bath. It is found that the system manifests a quantum decoherence which is more and more significant in time. We also calculate the decoherence time and show that it has the same scale as the time after which thermal fluctuations become comparable with quantum fluctuations. We solve the master equation for two independent harmonic oscillators interacting with an environment in the asymptotic long-time regime. We give a description of the continuous-variable asymptotic entanglement in terms of the covariance matrix of quantum states of the considered system for an arbitrary Gaussian input state. Using the Peres-Simon necessary and sufficient condition for separability of two-mode Gaussian states, we show that the two noninteracting systems immersed in a common environment become asymptotically entangled for certain environments, so that in the long-time regime they manifest nonlocal quantum correlations.
\end{abstract}

Keywords: open systems, quantum decoherence, quantum entanglement, nonseparable states.

\section{Introduction}

By quantum decoherence (QD) $[1,2]$ we understand the irreversible, uncontrollable, and persistent formation of quantum correlations (entanglement) of a system with its environment [3], expressed by the damping of the coherences present in the quantum state of the system, when off-diagonal elements of the density matrix decay below a certain level, so that this density matrix becomes approximately diagonal.

Since QD strongly depends on the interaction between the system and its external environment $[1,2]$, its role becomes relevant in many interesting physical problems. In many cases, one is interested in understanding QD because one wants to prevent decoherence from damaging quantum states and protect information stored in quantum states from degradation during the interaction with the environment. QD is also responsible for washing out the quantum interference effects which are desirable to be seen as signals in experiments, and it has a negative influence on many areas relying upon quantum coherence effects; in particular, QD is the main problem in quantum optics and physics of quantum information and computation [4].

Quantum entanglement represents the physical resource in quantum information science which is indispensable for the description and performance of such tasks like teleportation, superdense coding,

Manuscript submitted by the author in English on 13 August 2007.

1573-8760/07/2805-0439 ${ }^{\circledR} 2007$ Springer Science+Business Media, Inc. 
quantum cryptography, and quantum computation [4]. Therefore, the generation, detection, and manipulation of the entanglement continues to be presently a problem of intense investigation.

When two systems are immersed in an environment, then, in addition to and at the same time with the QD phenomenon, the external environment can also generate a quantum entanglement of the two systems and therefore an additional mechanism to correlate them [5-7]. In certain circumstances, the environment enhances the entanglement and in others it suppresses the entanglement, and the state describing the two systems becomes separable. The structure and properties of the environment may be such that not only the two systems become entangled, but also such that a certain amount of entanglement survives in the asymptotic long-time regime. The reason is that even if not directly coupled, the two systems immersed in the same environment can interact through the environment itself, and it depends on how strong this indirect interaction is with respect to the $\mathrm{QD}$, whether entanglement can be generated at the beginning of the evolution and, in the case of an affirmative answer, if it can be maintained for a definite time or it survives indefinitely in time [5].

In this paper, we study QD of a harmonic oscillator interacting with an environment, in particular with a thermal bath, within the framework of the theory of open quantum systems based on completely positive dynamical semigroups. We determine the degree of QD [8] for different regimes of the environment temperature. It is found that the system manifests a QD which, in general, increases with time and temperature. We also calculate the decoherence time and show that it has the same scale as the time after which thermal fluctuations become comparable with quantum fluctuations. Then we investigate, within the same framework, the existence of the continuous variable asymptotic entanglement for a subsystem composed of two identical harmonic oscillators interacting with an environment. We are interested in discussing the correlation effect of the environment; therefore, we assume that the two systems are independent, i.e., they do not interact directly. The initial state of the subsystem is taken of Gaussian form and the evolution under the quantum dynamical semigroup assures the preservation in time of the Gaussian form of the state. We only investigate here the asymptotic behavior of the subsystem states. The time evolution of the entanglement, in particular, the possibility of the so-called "entanglement sudden death," that is, suppression of the entanglement at a certain finite moment of time, will be discussed in a future work.

The paper is organized as follows.

In Sec. 2 we review the Markovian master equation for the damped harmonic oscillator and solve it in the coordinate representation. In Sec. 3 we investigate QD and calculate the decoherence time of the system. In Sec. 4 we write the equations of motion in the Heisenberg picture for two independent harmonic oscillators interacting with a general environment. With these equations, we derive in Sec. 5 the asymptotic values of the variances and covariances of the coordinates and momenta which enter the asymptotic covariance matrix. Then, by using the Peres-Simon necessary and sufficient condition for separability of two-mode Gaussian states $[9,10]$, we investigate the behavior of the environment-induced entanglement in the limit of long times. We show that for certain classes of environments the initial state evolves asymptotically to an equilibrium state which is entangled, while for other values of the parameters describing the environment, the entanglement is suppressed and the asymptotic state is separable. The existence of the quantum correlations between the two systems in the asymptotic long-time regime is the result of the competition between the entanglement and QD. A summary is given in Sec. 6 . 


\section{Markovian Master Equation for a Harmonic Oscillator}

In the axiomatic formalism based on completely positive quantum dynamical semigroups, the irreversible time evolution of an open system is described by the following general quantum Markovian master equation for the density operator $\rho(t)[11]$ :

$$
\frac{d \rho(t)}{d t}=-\frac{i}{\hbar}[H, \rho(t)]+\frac{1}{2 \hbar} \sum_{j}\left(\left[V_{j} \rho(t), V_{j}^{\dagger}\right]+\left[V_{j}, \rho(t) V_{j}^{\dagger}\right]\right) .
$$

The harmonic oscillator Hamiltonian $H$ is chosen in the general quadratic form:

$$
H=H_{0}+\frac{\mu}{2}\left(x p_{x}+p_{x} x\right), \quad H_{0}=\frac{1}{2 m} p_{x}^{2}+\frac{m \omega^{2}}{2} x^{2}
$$

and the operators $V_{j}$ and $V_{j}^{\dagger}$ modeling the environment are taken as linear polynomials in coordinate $x$ and momentum $p_{x}$. Then the master equation (1) takes the following form [12]:

$$
\begin{aligned}
\frac{d \rho}{d t}= & -\frac{i}{\hbar}\left[H_{0}, \rho\right]-\frac{i}{2 \hbar}(\lambda+\mu)\left[x, \rho p_{x}+p_{x} \rho\right]+\frac{i}{2 \hbar}(\lambda-\mu)\left[p_{x}, \rho x+x \rho\right] \\
& -\frac{D_{p_{x} p_{x}}}{\hbar^{2}}[x,[x, \rho]]-\frac{D_{x x}}{\hbar^{2}}\left[p_{x},\left[p_{x}, \rho\right]\right]+\left(\frac{D_{x p_{x}}}{\hbar^{2}}\left(\left[x,\left[p_{x}, \rho\right]\right]+\left[p_{x},[x, \rho]\right]\right) .\right.
\end{aligned}
$$

The diffusion coefficients $D_{x x}, D_{p_{x} p_{x}}$, and $D_{x p_{x}}$ and the dissipation constant $\lambda$ satisfy the fundamental constraints $D_{x x}>0, D_{p_{x} p_{x}}>0$, and

$$
D_{x x} D_{p_{x} p_{x}}-D_{x p_{x}}^{2} \geq \frac{\lambda^{2} \hbar^{2}}{4}
$$

In the particular case where the asymptotic state is a Gibbs state

$$
\rho_{G}(\infty)=\frac{e^{-H_{0} / k T}}{\operatorname{Tr} e^{-H_{0} / k T}}
$$

these coefficients become

$$
D_{x x}=\frac{\lambda-\mu}{2} \frac{\hbar}{m \omega} \operatorname{coth} \frac{\hbar \omega}{2 k T}, \quad D_{p_{x} p_{x}}=\frac{\lambda+\mu}{2} \hbar m \omega \operatorname{coth} \frac{\hbar \omega}{2 k T}, \quad D_{x p_{x}}=0,
$$

where $T$ is the temperature of the thermal bath. In this case, the fundamental constraints are satisfied only if $\lambda>\mu$ and

$$
\left(\lambda^{2}-\mu^{2}\right) \operatorname{coth}^{2} \frac{\hbar \omega}{2 k T} \geq \lambda^{2}
$$

and the asymptotic values $\sigma_{x x}(\infty), \sigma_{p_{x} p_{x}}(\infty)$, and $\sigma_{x p_{x}}(\infty)$ of the dispersion (variance) and, correspondingly, the correlation (covariance) of the coordinate and momentum reduce to [12]:

$$
\sigma_{x x}(\infty)=\frac{\hbar}{2 m \omega} \operatorname{coth} \frac{\hbar \omega}{2 k T}, \quad \sigma_{p_{x} p_{x}}(\infty)=\frac{\hbar m \omega}{2} \operatorname{coth} \frac{\hbar \omega}{2 k T}, \quad \sigma_{x p_{x}}(\infty)=0 .
$$


We consider a harmonic oscillator with an initial Gaussian wave function $\left[\sigma_{x}(0)\right.$ and $\sigma_{p_{x}}(0)$ are the initial averaged position and momentum of the wave packet]

$$
\Psi(x)=\left(\frac{1}{2 \pi \sigma_{x x}(0)}\right)^{1 / 4} \exp \left[-\frac{1}{4 \sigma_{x x}(0)}\left(1-\frac{2 i}{\hbar} \sigma_{x p_{x}}(0)\right)\left(x-\sigma_{x}(0)\right)^{2}+\frac{i}{\hbar} \sigma_{p_{x}}(0) x\right]
$$

representing a correlated coherent state [13] (squeezed coherent state) with the variances and covariance of coordinate and momentum

$$
\sigma_{x x}(0)=\frac{\hbar \delta}{2 m \omega}, \quad \sigma_{p_{x} p_{x}}(0)=\frac{\hbar m \omega}{2 \delta\left(1-r^{2}\right)}, \quad \sigma_{x p_{x}}(0)=\frac{\hbar r}{2 \sqrt{1-r^{2}}} .
$$

Here $\delta$ is the squeezing parameter that measures the spread in the initial Gaussian packet, and $r$ (with $|r|<1$ ) is the correlation coefficient. The initial values (9) correspond to the minimum uncertainty state, since they fulfill the generalized uncertainty relation

$$
\sigma_{x x}(0) \sigma_{p_{x} p_{x}}(0)-\sigma_{x p_{x}}^{2}(0)=\frac{\hbar^{2}}{4}
$$

For $\delta=1$ and $r=0$, the correlated coherent state becomes the Glauber coherent state.

From Eq. (3) we derive the evolution equation in the coordinate representation

$$
\begin{aligned}
\frac{\partial \rho}{\partial t}= & \frac{i \hbar}{2 m}\left(\frac{\partial^{2}}{\partial x^{2}}-\frac{\partial^{2}}{\partial x^{\prime 2}}\right) \rho-\frac{i m \omega^{2}}{2 \hbar}\left(x^{2}-x^{\prime 2}\right) \rho \\
& -\frac{1}{2}(\lambda+\mu)\left(x-x^{\prime}\right)\left(\frac{\partial}{\partial x}-\frac{\partial}{\partial x^{\prime}}\right) \rho+\frac{1}{2}(\lambda-\mu)\left[\left(x+x^{\prime}\right)\left(\frac{\partial}{\partial x}+\frac{\partial}{\partial x^{\prime}}\right)+2\right] \rho \\
& -\frac{D_{p_{x} p_{x}}}{\hbar^{2}}\left(x-x^{\prime}\right)^{2} \rho+D_{x x}\left(\frac{\partial}{\partial x}+\frac{\partial}{\partial x^{\prime}}\right)^{2} \rho-2 i D_{x p_{x}} \hbar\left(x-x^{\prime}\right)\left(\frac{\partial}{\partial x}+\frac{\partial}{\partial x^{\prime}}\right) \rho .
\end{aligned}
$$

The first two terms on the right-hand side of this equation generate the usual Liouvillian unitary evolution. The third and fourth terms are the dissipative terms and have a damping effect (exchange of energy with environment). The last three are noise (diffusive) terms and produce fluctuation effects in the evolution of the system. $D_{p_{x} p_{x}}$ promotes diffusion in momentum and generates decoherence in coordinate $x$ - it reduces the off-diagonal terms responsible for correlations between spatially separated pieces of the wave packet. Similarly $D_{x x}$ promotes diffusion in coordinate and generates decoherence in momentum $p_{x}$. The $D_{x p_{x}}$ term is the so-called "anomalous diffusion" term and it does not generate decoherence.

The density matrix solution of Eq. (11) has the general Gaussian form

$$
\begin{aligned}
\left\langle x|\rho(t)| x^{\prime}\right\rangle= & \left(\frac{1}{2 \pi \sigma_{x x}(t)}\right)^{1 / 2} \exp \left[-\frac{1}{2 \sigma_{x x}(t)}\left(\frac{x+x^{\prime}}{2}-\sigma_{x}(t)\right)^{2}-\frac{\sigma(t)}{2 \hbar^{2} \sigma_{x x}(t)}\left(x-x^{\prime}\right)^{2}\right. \\
& \left.+\frac{i \sigma_{x p_{x}}(t)}{\hbar \sigma_{x x}(t)}\left(\frac{x+x^{\prime}}{2}-\sigma_{x}(t)\right)\left(x-x^{\prime}\right)+\frac{i}{\hbar} \sigma_{p_{x}}(t)\left(x-x^{\prime}\right)\right],
\end{aligned}
$$

where

$$
\sigma(t) \equiv \sigma_{x x}(t) \sigma_{p_{x} p_{x}}(t)-\sigma_{x p_{x}}^{2}(t)
$$


is the determinant of the covariance matrix

$$
\left(\begin{array}{cc}
\sigma_{x x}(t) & \sigma_{x p_{x}}(t) \\
\sigma_{x p_{x}}(t) & \sigma_{p_{x} p_{x}}(t)
\end{array}\right)
$$

and represents also the Schrödinger generalized uncertainty function.

In the case of a thermal bath, we obtain the following stationary state solution for $t \rightarrow \infty$ $(\epsilon \equiv \hbar \omega / 2 k T)$ :

$$
\left\langle x|\rho(\infty)| x^{\prime}\right\rangle=\left(\frac{m \omega}{\pi \hbar \operatorname{coth} \epsilon}\right)^{1 / 2} \exp \left\{-\frac{m \omega}{4 \hbar}\left[\frac{\left(x+x^{\prime}\right)^{2}}{\operatorname{coth} \epsilon}+\left(x-x^{\prime}\right)^{2} \operatorname{coth} \epsilon\right]\right\} .
$$

\section{Quantum Decoherence}

An isolated system has a unitary evolution and the coherence of the state is not lost - pure states evolve in time only to pure states. The QD phenomenon, that is, the loss of coherence or the destruction of off-diagonal elements representing coherences between quantum states in the density matrix, can be achieved by introducing an interaction between the system and environment - an initial pure state with a density matrix which contains nonzero off-diagonal terms can nonunitarily evolve into a final mixed state with a diagonal density matrix.

In the literature, several measures of the degree of decoherence have been introduced (expressed in terms of the von Neumann entropy [14], linear entropy [15,16], or the coefficients of the matrix elements of the statistical operator with respect to the energy basis [17]).

Using new variables $\Sigma=\left(x+x^{\prime}\right) / 2$ and $\Delta=x-x^{\prime}$, the density matrix (12) becomes

$$
\rho(\Sigma, \Delta, t)=\sqrt{\frac{\alpha}{\pi}} \exp \left[-\alpha \Sigma^{2}-\gamma \Delta^{2}+i \beta \Sigma \Delta+2 \alpha \sigma_{x}(t) \Sigma+i\left(\frac{\sigma_{p_{x}}(t)}{\hbar}-\beta \sigma_{x}(t)\right) \Delta-\alpha \sigma_{x}^{2}(t)\right],
$$

with the abbreviations

$$
\alpha=\frac{1}{2 \sigma_{x x}(t)}, \quad \gamma=\frac{\sigma(t)}{2 \hbar^{2} \sigma_{x x}(t)}, \quad \beta=\frac{\sigma_{x p_{x}}(t)}{\hbar \sigma_{x x}(t)} .
$$

Here we use the representation-independent measure of the degree of QD [8], which is given by the ratio

of the dispersion $1 / \sqrt{2 \gamma}$ of the off-diagonal element $\rho(0, \Delta, t)$ to the dispersion $\sqrt{2 / \alpha}$ of the diagonal element $\rho(\Sigma, 0, t)$, namely,

$$
\delta_{Q D}(t)=\frac{1}{2} \sqrt{\frac{\alpha}{\gamma}}=\frac{\hbar}{2 \sqrt{\sigma(t)}} .
$$

It can be easily shown that $\delta_{Q D}$ is related to the linear entropy [18].

The finite-temperature Schrödinger generalized uncertainty function has the expression [19] (with the notation $\left.\Omega^{2} \equiv \omega^{2}-\mu^{2}, \omega>\mu\right)$

$$
\begin{aligned}
\sigma(t)= & \frac{\hbar^{2}}{4}\left\{e^{-4 \lambda t}\left[1-\left(\delta+\frac{1}{\delta\left(1-r^{2}\right)}\right) \operatorname{coth} \epsilon+\operatorname{coth}^{2} \epsilon\right]\right. \\
& +e^{-2 \lambda t} \operatorname{coth} \epsilon\left[\left(\delta+\frac{1}{\delta\left(1-r^{2}\right)}-2 \operatorname{coth} \epsilon\right) \frac{\omega^{2}-\mu^{2} \cos (2 \Omega t)}{\Omega^{2}}\right. \\
& \left.\left.+\left(\delta-\frac{1}{\delta\left(1-r^{2}\right)}\right) \frac{\mu \sin (2 \Omega t)}{\Omega}+\frac{2 r \mu \omega[1-\cos (2 \Omega t)]}{\Omega^{2} \sqrt{1-r^{2}}}\right]+\operatorname{coth}^{2} \epsilon\right\} .
\end{aligned}
$$


In the limit of long times, Eq. (19) yields

$$
\sigma(\infty)=\frac{\hbar^{2}}{4} \operatorname{coth}^{2} \epsilon
$$

so that we obtain

$$
\delta_{Q D}(\infty)=\tanh \frac{\hbar \omega}{2 k T},
$$

which for high $T$ becomes

$$
\delta_{Q D}(\infty)=\frac{\hbar \omega}{2 k T} .
$$

Figure 1 illustrates the dependence of the QD degree on temperature and time. We see that, in general, $\delta_{Q D}$ decreases and, therefore, QD becomes stronger with increasing time and temperature, i.e., the density matrix becomes more and more diagonal at higher $T$ and the contributions of the off-diagonal elements get smaller and smaller. At the same time, the degree of purity decreases and the degree of mixedness increases with $T$. For $T=0$, the asymptotic (final) state is pure and $\delta_{Q D}$ reaches its initial maximum value $1 ; \delta_{Q D}=0$ when the quantum coherence is completely lost and, if $\delta_{Q D}=1$, there is no QD. Only if $\delta_{Q D}<1$ can we say that the considered system interacting with the thermal bath manifests $\mathrm{QD}$, when the value of the elements of

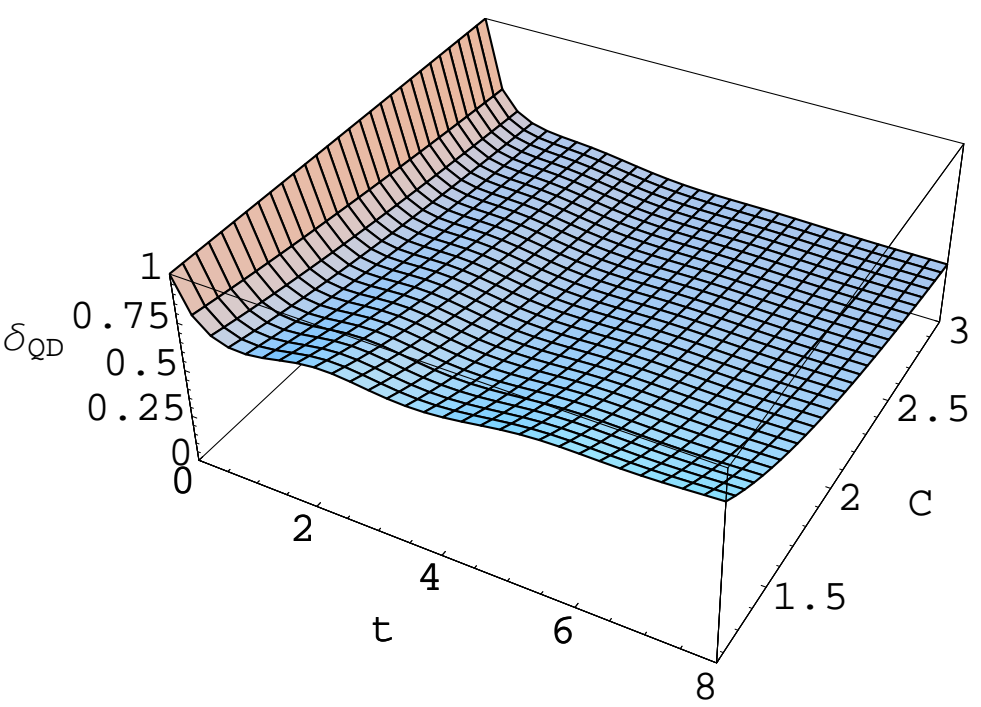

Fig. 1. Degree of quantum decoherence $\delta_{Q D}$ versus temperature $T$ [through $C \equiv \operatorname{coth}(\hbar \omega / 2 k T)]$ and time $t$ for $\lambda=0.2, \mu=0.1, \delta=4$, and $r=0$.

the density matrix in the position basis are peaked preferentially along the diagonal $x=x^{\prime}$. In Fig. 2 the density matrix in the coordinate representation (12) at the initial and final moments of time is presented. The values of the elements of the density matrix along the diagonal $x=x^{\prime}$ represent the probability of finding the system in this position, while values of off-diagonal elements represent the correlations in the density matrix between the points $x$ and $x^{\prime}$. For simplicity, in Fig. 2 we consider zero values for the initial expectations values of coordinate and momentum, so that the density matrix is centered in origin. Dissipation promotes quantum coherence, whereas fluctuation (diffusion) reduces coherence and promotes QD. The balance of dissipation and fluctuation determines the final equilibrium value of $\delta_{Q D}[20]$.

In order to obtain the expression of the decoherence time, we consider the coefficient $\gamma(17)$, which measures the contribution of nondiagonal elements in the density matrix (16). For short times $(\lambda t \ll$ $1, \Omega t \ll 1)$, we have

$$
\gamma(t)=-\frac{m \omega}{4 \hbar \delta}\left\{1+2\left[\lambda\left(\delta+\frac{r^{2}}{\delta\left(1-r^{2}\right)}\right) \operatorname{coth} \epsilon+\mu\left(\delta-\frac{r^{2}}{\delta\left(1-r^{2}\right)}\right) \operatorname{coth} \epsilon-\lambda-\mu-\frac{\omega r}{\delta \sqrt{1-r^{2}}}\right] t\right\} .
$$



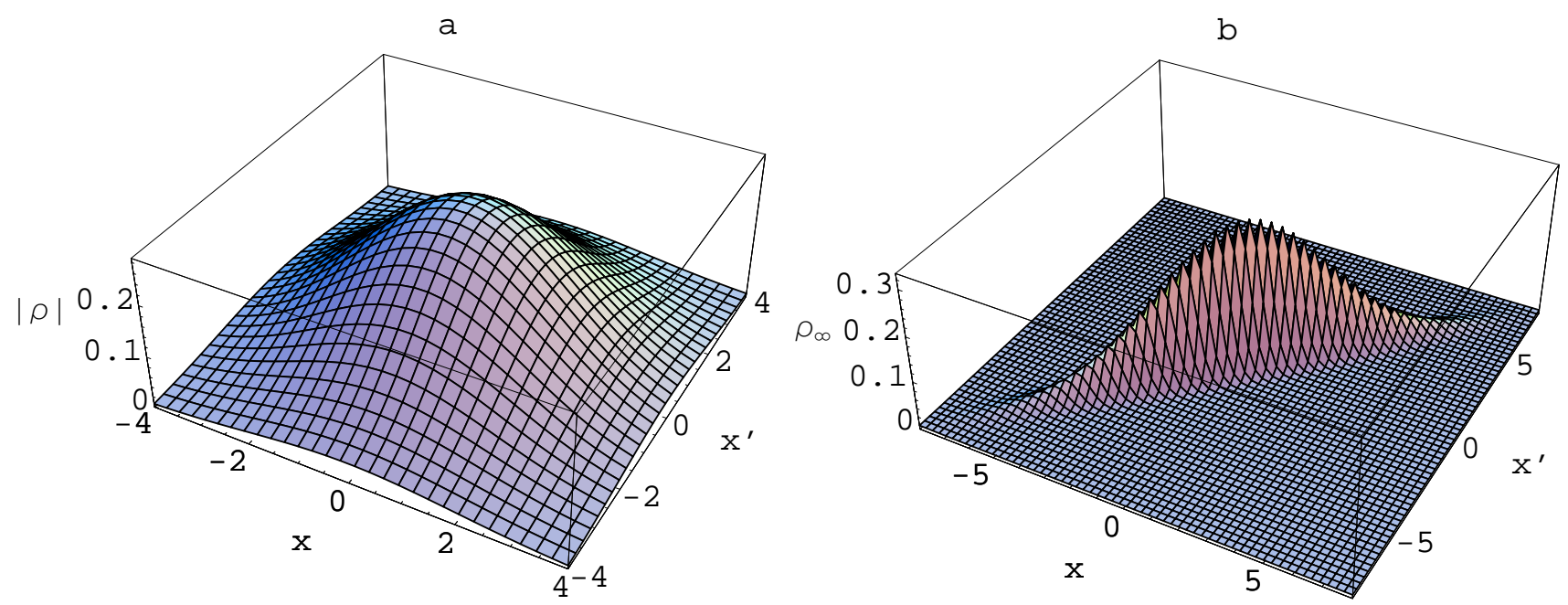

Fig. 2. Density matrix $\rho$ in the coordinate representation for $\lambda=0.2, \mu=0.1, \delta=4$, and $r=0$ : $|\rho|$ at the initial time $t=0$ (a) and $\rho_{\infty}$ for $C=10(\mathrm{~b})$.

From here we conclude that the quantum coherence in the density matrix decays exponentially and the time scale for decoherence is given by

$$
t_{\mathrm{deco}}=\left\{2\left[\lambda\left(\delta+\frac{r^{2}}{\delta\left(1-r^{2}\right)}\right) \operatorname{coth} \epsilon+\mu\left(\delta-\frac{r^{2}}{\delta\left(1-r^{2}\right)}\right) \operatorname{coth} \epsilon-\lambda-\mu-\frac{\omega r}{\delta \sqrt{1-r^{2}}}\right]\right\}^{-1} .
$$

The decoherence time depends on temperature $T$ and coupling $\lambda$ (dissipation coefficient) between the system and environment, as well as on the squeezing parameter $\delta$ and initial correlation coefficient $r$. We notice that the decoherence time is decreasing with increasing dissipation, temperature, and squeezing.

For $r=0$, we obtain

$$
t_{\mathrm{deco}}=\frac{1}{2(\lambda+\mu)(\delta \operatorname{coth} \epsilon-1)}
$$

and at temperature $T=0$ [here we have to take $\mu=0$ due to Eq. (6)], this becomes

$$
t_{\mathrm{deco}}=\frac{1}{2 \lambda(\delta-1)}
$$

We see that when the initial state is the usual coherent state $(\delta=1)$, the decoherence time tends to infinity. This corresponds to the fact that for $T=0$ and $\delta=1$ the coefficient $\gamma$ is constant in time, so that the decoherence process does not occur in this case.

At high temperatures, expression (24) becomes

$$
t_{\mathrm{deco}}=\left\{2\left[\lambda\left(\delta+\frac{r^{2}}{\delta\left(1-r^{2}\right)}\right)+\mu\left(\delta-\frac{r^{2}}{\delta\left(1-r^{2}\right)}\right)\right] \frac{2 k T}{\hbar \omega}\right\}^{-1} .
$$

If, in addition, $r=0$, we obtain

$$
t_{\mathrm{deco}}=\frac{\hbar \omega}{4(\lambda+\mu) \delta k T} .
$$


The generalized uncertainty function $\sigma(t)(19)$ has the following behavior at short times:

$$
\sigma(t)=\frac{\hbar^{2}}{4}\left\{1+2\left[\lambda\left(\delta+\frac{1}{\delta\left(1-r^{2}\right)}\right) \operatorname{coth} \epsilon+\mu\left(\delta-\frac{1}{\delta\left(1-r^{2}\right)}\right) \operatorname{coth} \epsilon-2 \lambda\right] t\right\} .
$$

This expression shows explicitly the contribution for small time of uncertainty that is intrinsic to quantum mechanics, expressed through the Heisenberg uncertainty principle and uncertainty due to the coupling to the thermal environment. From Eq. (29) we can determine time $t_{d}$ when thermal fluctuations become comparable with quantum fluctuations. At high temperatures, we obtain

$$
t_{d}=\left\{2\left[\lambda\left(\delta+\frac{1}{\delta\left(1-r^{2}\right)}\right)+\mu\left(\delta-\frac{1}{\delta\left(1-r^{2}\right)}\right)\right] \frac{2 k T}{\hbar \omega}\right\}^{-1} .
$$

As expected, the decoherence time $t_{\text {deco }}$ has the same scale as time $t_{d}$ after which thermal fluctuations become comparable with quantum fluctuations [20,21]. The values of $t_{\text {deco }}$ and $t_{d}$ become closer with increasing temperature and squeezing.

When $t \gg t_{\text {rel }}$, where $t_{\text {rel }} \approx \lambda^{-1}$ is the relaxation time, which governs the rate of energy dissipation, the particle reaches equilibrium with the environment. In the macroscopic domain, QD occurs very much faster than relaxation, so that for all macroscopic bodies the dissipation term becomes important much later after the decoherence term has already dominated and diminished the off-diagonal terms of the density matrix. We remark also that $t_{\text {deco }}$ can be of the order of $t_{\text {rel }}$ for sufficiently low temperatures and small wave packet spread (small squeezing coefficient).

\section{Equations of Motion for Two Independent Harmonic Oscillators}

Now we are interested in the generation of entanglement between two harmonic oscillators due to the back-action of the environment on the subsystem. Since the two harmonic oscillators interact with a common environment, the coupling between the two oscillators will be induced even when initially they are uncoupled. Thus, the master equation for the two harmonic oscillators must take into account their mutual interaction by their coupling to the environment. We study the dynamics of the subsystem composed of two identical noninteracting (independent) oscillators in weak interaction with a large environment, so that their reduced time evolution can be described by the Markovian completely positive quantum dynamical semigroup like in the previous case of one harmonic oscillator.

If $\widetilde{\Phi}_{t}$ is the dynamical semigroup describing the time evolution of open quantum system in the Heisenberg picture $\left[\widetilde{\Phi}_{t}\right.$ is the dual of the dynamical semigroup $\Phi_{t}$ which describes the time evolution in the Schrödinger picture given by Eq. (1)], then the master equation is given for an operator $A$ as follows $[11,12]$ :

$$
\frac{d \widetilde{\Phi}_{t}(A)}{d t}=\frac{i}{\hbar}\left[H, \widetilde{\Phi}_{t}(A)\right]+\frac{1}{2 \hbar} \sum_{j}\left(V_{j}^{\dagger}\left[\widetilde{\Phi}_{t}(A), V_{j}\right]+\left[V_{j}^{\dagger}, \widetilde{\Phi}_{t}(A)\right] V_{j}\right)
$$

Here, again $H$ denotes the Hamiltonian of the open quantum system, and $V_{j}$ and $V_{j}^{\dagger}$, being operators defined on the Hilbert space of $H$, represent the interaction of the open system with the environment. Being interested in the set of Gaussian states, we introduce those quantum dynamical semigroups that preserve that set. Therefore, $H$ is taken to be a polynomial of second degree in the coordinates $x, y$ and 
momenta $p_{x}, p_{y}$ of the two quantum oscillators, and $V_{j}, V_{j}^{\dagger}$ are taken to be polynomials of only first degree in these canonical observables. Then, in the linear space spanned by the coordinates and momenta, there exist only four linearly independent operators $V_{j=1,2,3,4}[22]$ :

$$
V_{j}=a_{x j} p_{x}+a_{y j} p_{y}+b_{x j} x+b_{y j} y
$$

where $a_{x j}, a_{y j}, b_{x j}, b_{y j} \in \mathbf{C}$ and

$$
V_{j}^{\dagger}=a_{x j}^{*} p_{x}+a_{y j}^{*} p_{y}+b_{x j}^{*} x+b_{y j}^{*} y
$$

(the star denotes complex conjugation). The Hamiltonian $H$ of the two uncoupled identical harmonic oscillators of mass $m$ and frequency $\omega$ is given as follows:

$$
H=\frac{1}{2 m}\left(p_{x}^{2}+p_{y}^{2}\right)+\frac{m \omega^{2}}{2}\left(x^{2}+y^{2}\right) .
$$

The fact that $\widetilde{\Phi}_{t}$ is a dynamical semigroup implies the positivity of the following matrix formed by the scalar products of the four vectors $\mathbf{a}_{x}, \mathbf{b}_{x}, \mathbf{a}_{y}$, and $\mathbf{b}_{y}$ whose entries are the components $a_{x j}, a_{y j}, b_{x j}$, and $b_{y j}$, respectively,

$$
\frac{1}{2} \hbar\left(\begin{array}{rrrr}
\left(\mathbf{a}_{x} \mathbf{a}_{x}\right) & \left(\mathbf{a}_{x} \mathbf{b}_{x}\right) & \left(\mathbf{a}_{x} \mathbf{a}_{y}\right) & \left(\mathbf{a}_{x} \mathbf{b}_{y}\right) \\
\left(\mathbf{b}_{x} \mathbf{a}_{x}\right) & \left(\mathbf{b}_{x} \mathbf{b}_{x}\right) & \left(\mathbf{b}_{x} \mathbf{a}_{y}\right) & \left(\mathbf{b}_{x} \mathbf{b}_{y}\right) \\
\left(\mathbf{a}_{y} \mathbf{a}_{x}\right) & \left(\mathbf{a}_{y} \mathbf{b}_{x}\right) & \left(\mathbf{a}_{y} \mathbf{a}_{y}\right) & \left(\mathbf{a}_{y} \mathbf{b}_{y}\right) \\
\left(\mathbf{b}_{y} \mathbf{a}_{x}\right) & \left(\mathbf{b}_{y} \mathbf{b}_{x}\right) & \left(\mathbf{b}_{y} \mathbf{a}_{y}\right) & \left(\mathbf{b}_{y} \mathbf{b}_{y}\right)
\end{array}\right)
$$

For simplicity, we take this matrix of the following form, where all coefficients $D_{x x}, D_{x p_{x}}, \ldots$ and $\lambda$ are real quantities:

$$
\left(\begin{array}{cccc}
D_{x x} & -D_{x p_{x}}-i \hbar \lambda / 2 & D_{x y} & -D_{x p_{y}} \\
-D_{x p_{x}}+i \hbar \lambda / 2 & D_{p_{x} p_{x}} & -D_{y p_{x}} & D_{p_{x} p_{y}} \\
D_{x y} & -D_{y p_{x}} & D_{y y} & -D_{y p_{y}}-i \hbar \lambda / 2 \\
-D_{x p_{y}} & D_{p_{x} p_{y}} & -D_{y p_{y}}+i \hbar \lambda / 2 & D_{p_{y} p_{y}}
\end{array}\right) \text {. }
$$

It follows that the principal minors of this matrix are positive or zero. From the Cauchy-Schwarz inequality, the following relations for the coefficients defined in Eq. (36) hold (here and below, we put, for simplicity, $\hbar=1)$ :

$$
\begin{array}{r}
D_{x x} D_{y y}-D_{x y}^{2} \geq 0, \quad D_{x x} D_{p_{x} p_{x}}-D_{x p_{x}}^{2} \geq \frac{\lambda^{2}}{4}, \quad D_{x x} D_{p_{y} p_{y}}-D_{x p_{y}}^{2} \geq 0, \\
D_{y y} D_{p_{x} p_{x}}-D_{y p_{x}}^{2} \geq 0, \quad D_{y y} D_{p_{y} p_{y}}-D_{y p_{y}}^{2} \geq \frac{\lambda^{2}}{4}, \quad D_{p_{x} p_{x}} D_{p_{y} p_{y}}-D_{p_{x} p_{y}}^{2} \geq 0 .
\end{array}
$$

The matrix of the coefficients (36) can be conveniently written as

$$
\left(\begin{array}{cc}
C_{1} & C_{3} \\
C_{3}^{\dagger} & C_{2}
\end{array}\right)
$$


in terms of $2 \times 2$ matrices $C_{1}=C_{1}^{\dagger}, C_{2}=C_{2}^{\dagger}$, and $C_{3}$. This decomposition has a direct physical interpretation - the elements containing the diagonal contributions $C_{1}$ and $C_{2}$ represent diffusion and dissipation coefficients corresponding to the first (second) system in the absence of the other, while the elements in $C_{3}$ represent environment generated couplings between the two initially-independent oscillators.

The variance and covariance of self-adjoint operators $A_{1}$ and $A_{2}$ can be written with the density operator $\rho$, describing the initial state of the quantum system, as follows:

$$
\sigma_{A_{1} A_{2}}(t)=\frac{1}{2} \operatorname{Tr}\left[\rho \widetilde{\Phi}_{t}\left(A_{1} A_{2}+A_{2} A_{1}\right)\right] .
$$

We introduce the following $4 \times 4$ covariance matrix:

$$
\sigma(t)=\left(\begin{array}{cccc}
\sigma_{x x} & \sigma_{x p_{x}} & \sigma_{x y} & \sigma_{x p_{y}} \\
\sigma_{x p_{x}} & \sigma_{p_{x} p_{x}} & \sigma_{y p_{x}} & \sigma_{p_{x} p_{y}} \\
\sigma_{x y} & \sigma_{y p_{x}} & \sigma_{y y} & \sigma_{y p_{y}} \\
\sigma_{x p_{y}} & \sigma_{p_{x} p_{y}} & \sigma_{y p_{y}} & \sigma_{p_{y} p_{y}}
\end{array}\right) .
$$

By direct calculation, we obtain [22]

$$
\frac{d \sigma}{d t}=Y \sigma+\sigma Y^{\mathrm{T}}+2 D
$$

where

$$
Y=\left(\begin{array}{cccc}
-\lambda & 1 / m & 0 & 0 \\
-m \omega^{2} & -\lambda & 0 & 0 \\
0 & 0 & -\lambda & 1 / m \\
0 & 0 & -m \omega^{2} & -\lambda
\end{array}\right)
$$

$D$ is the matrix of the diffusion coefficients

$$
D=\left(\begin{array}{cccc}
D_{x x} & D_{x p_{x}} & D_{x y} & D_{x p_{y}} \\
D_{x p_{x}} & D_{p_{x} p_{x}} & D_{y p_{x}} & D_{p_{x} p_{y}} \\
D_{x y} & D_{y p_{x}} & D_{y y} & D_{y p_{y}} \\
D_{x p_{y}} & D_{p_{x} p_{y}} & D_{y p_{y}} & D_{p_{y} p_{y}}
\end{array}\right),
$$

and $Y^{\mathrm{T}}$ is the transposed matrix of $Y$.

The time-dependent solution of Eq. (42) is given by [22]

$$
\sigma(t)=M(t)[\sigma(0)-\sigma(\infty)] M^{\mathrm{T}}(t)+\sigma(\infty),
$$

where $M(t)=e^{t Y}$. The matrix $M(t)$ has to fulfill the condition $\lim _{t \rightarrow \infty} M(t)=0$. In order that this limit exists, $Y$ must only have eigenvalues with negative real parts. The values at infinity are obtained from the equation $[22]$

$$
Y \sigma(\infty)+\sigma(\infty) Y^{\mathrm{T}}=-2 D
$$




\section{Environment-Induced Entanglement}

The two-mode Gaussian state is entirely specified by its covariance matrix $\sigma$ (41), which is a real, symmetric, and positive matrix with the following block structure:

$$
\sigma=\left(\begin{array}{cc}
A & C \\
C^{\mathrm{T}} & B
\end{array}\right),
$$

where $A, B$, and $C$ are $2 \times 2$ matrices. Their entries are correlations of the canonical operators $x, y, p_{x}$, and $p_{y}, A$ and $B$ denote the symmetric covariance matrices for the individual reduced one-mode states, while the matrix $C$ contains the cross-correlations between modes. The entries of the covariance matrix depend on $Y$ and $D$ and can be calculated from Eq. (46). To simplify further the calculations, we consider environments for which the two diagonal submatrices in Eq. (39) are equal $\left(C_{1}=C_{2}\right)$, so that $D_{x x}=D_{y y}, D_{x p_{x}}=D_{y p_{y}}$, and $D_{p_{x} p_{x}}=D_{p_{y} p_{y}}$.

In addition, in the matrix $C_{3}$ we take $D_{x p_{y}}=D_{y p_{x}}$.

Then both unimodal covariance matrices are equal $(A=B)$ and the entanglement matrix $C$ is symmetric. With the chosen coefficients, we obtain the following elements of the asymptotic entanglement matrix $C$ :

$$
\begin{gathered}
\sigma_{x y}(\infty)=\frac{m^{2}\left(2 \lambda^{2}+\omega^{2}\right) D_{x y}+2 m \lambda D_{x p_{y}}+D_{p_{x} p_{y}}}{2 m^{2} \lambda\left(\lambda^{2}+\omega^{2}\right)}, \\
\sigma_{x p_{y}}(\infty)=\sigma_{y p_{x}}(\infty)=\frac{-m^{2} \omega^{2} D_{x y}+2 m \lambda D_{x p_{y}}+D_{p_{x} p_{y}}}{2 m\left(\lambda^{2}+\omega^{2}\right)}, \\
\sigma_{p_{x} p_{y}}(\infty)=\frac{m^{2} \omega^{4} D_{x y}-2 m \omega^{2} \lambda D_{x p_{y}}+\left(2 \lambda^{2}+\omega^{2}\right) D_{p_{x} p_{y}}}{2 \lambda\left(\lambda^{2}+\omega^{2}\right)},
\end{gathered}
$$

and the following elements of the matrices $A$ and $B$ :

$$
\begin{gathered}
\sigma_{x x}(\infty)=\sigma_{y y}(\infty)=\frac{m^{2}\left(2 \lambda^{2}+\omega^{2}\right) D_{x x}+2 m \lambda D_{x p_{x}}+D_{p_{x} p_{x}}}{2 m^{2} \lambda\left(\lambda^{2}+\omega^{2}\right)}, \\
\sigma_{x p_{x}}(\infty)=\sigma_{y p_{y}}(\infty)=\frac{-m^{2} \omega^{2} D_{x x}+2 m \lambda D_{x p_{x}}+D_{p_{x} p_{x}}}{2 m\left(\lambda^{2}+\omega^{2}\right)} \\
\sigma_{p_{x} p_{x}}(\infty)=\sigma_{p_{y} p_{y}}(\infty)=\frac{m^{2} \omega^{4} D_{x x}-2 m \omega^{2} \lambda D_{x p_{x}}+\left(2 \lambda^{2}+\omega^{2}\right) D_{p_{x} p_{x}}}{2 \lambda\left(\lambda^{2}+\omega^{2}\right)}
\end{gathered}
$$

With these quantities, we calculate the determinant of the entanglement matrix

$$
\operatorname{det} C=\frac{1}{4 \lambda^{2}\left(\lambda^{2}+\omega^{2}\right)}\left[\left(m \omega^{2} D_{x y}+\frac{1}{m} D_{p_{x} p_{y}}\right)^{2}+4 \lambda^{2}\left(D_{x y} D_{p_{x} p_{y}}-D_{x p_{y}}^{2}\right)\right] .
$$


It is very interesting that the general theory of open quantum systems allows couplings through the environment between uncoupled oscillators. According to the definition of the environment parameters, the diffusion coefficients above can be different from zero and can simulate the interaction between uncoupled oscillators. Indeed, the Gaussian states with $\operatorname{det} C \geq 0$ are separable states but, for $\operatorname{det} C<0$, it may be possible that the asymptotic equilibrium states are entangled, as will be shown below.

On general grounds, one expects that the effects of decoherence, counteracting the entanglement production, are dominant in the long-time regime, so that no quantum correlation (entanglement) is expected to be left at infinity. Nevertheless, there are situations where the environment allows the presence of entangled asymptotic equilibrium states. In order to investigate whether an external environment can actually entangle the two independent systems, we can use the partial transposition criterion $[9,10]$ - a state becomes entangled if and only if the operation of partial transposition does not preserve its positivity. Simon [10] obtained the necessary and sufficient criterion for separability $S \geq 0$, where

$$
S \equiv \operatorname{det} A \operatorname{det} B+\left(\frac{1}{4}-|\operatorname{det} C|\right)^{2}-\operatorname{Tr}\left[A J C J B J C^{\mathrm{T}} J\right]-\frac{1}{4}(\operatorname{det} A+\operatorname{det} B)
$$

and $J$ is the $2 \times 2$ symplectic matrix

$$
J=\left(\begin{array}{cc}
0 & 1 \\
-1 & 0
\end{array}\right)
$$

In order to analyze the possible persistence of environment-induced entanglement in the asymptotic long-time regime, we consider the environment characterized by the following values of its parameters:

$$
m^{2} \omega^{2} D_{x x}=D_{p_{x} p_{x}}, \quad D_{x p_{x}}=0, \quad m^{2} \omega^{2} D_{x y}=D_{p_{x} p_{y}} .
$$

In this case, the Simon expression (55) takes the form

$$
S=\left(\frac{m^{2} \omega^{2}\left(D_{x x}^{2}-D_{x y}^{2}\right)}{\lambda^{2}}+\frac{D_{x p_{y}}^{2}}{\lambda^{2}+\omega^{2}}-\frac{1}{4}\right)^{2}-4 \frac{m^{2} \omega^{2} D_{x x}^{2} D_{x p_{y}}^{2}}{\lambda^{2}\left(\lambda^{2}+\omega^{2}\right)} .
$$

For environments characterized by coefficients such that expression (57) is negative, the asymptotic final state is entangled. In particular, if $D_{x y}=0$, we obtain $S<0$, i.e., the asymptotic final state is entangled for the following range of values of the coefficient $D_{x p_{y}}$ characterizing the environment:

$$
\frac{m \omega D_{x x}}{\lambda}-\frac{1}{2}<\frac{D_{x p_{y}}}{\sqrt{\lambda^{2}+\omega^{2}}}<\frac{m \omega D_{x x}}{\lambda}+\frac{1}{2}
$$

where the coefficient $D_{x x}$ satisfies the condition $m \omega D_{x x} / \lambda \geq 1 / 2$, equivalent to the unimodal uncertainty relation. If the coefficients do not fulfil inequalities (58), then $S \geq 0$ and, therefore, the asymptotic final state of the considered bipartite system is separable.

\section{Summary}

We have studied QD with the Markovian equation of Lindblad for a system consisting of an onedimensional harmonic oscillator in interaction with a thermal bath within the framework of the theory 
of open quantum systems based on completely positive quantum dynamical semigroups. Within the same framework, we investigated the existence of the asymptotic quantum entanglement for a subsystem composed of two uncoupled identical harmonic oscillators interacting with an environment.

(i) We have shown that $\mathrm{QD}$, in general, increases with time and temperature. For large temperatures, QD is strong and the degree of mixedness is high, while for zero temperature the asymptotic final state is pure. With increasing squeezing parameter and initial correlation, QD becomes stronger but the asymptotic value of the degree of QD does not depend on the initial squeezing and correlation; it depends on temperature only. QD is expressed by the loss of quantum coherences in the case of a thermal bath at finite temperature.

(ii) We determined the general expression of the decoherence time, which shows that it is decreasing with increasing dissipation, temperature, and squeezing. We have also shown that the decoherence time has the same scale as the time after which thermal fluctuations become comparable with quantum fluctuations and the values of these scales become closer with increasing temperature and squeezing.

(iii) By using the Peres-Simon necessary and sufficient condition for separability of two-mode Gaussian states, we have shown that for certain classes of environments the initial state evolves asymptotically to an equilibrium state which is entangled, i.e., there exist nonlocal quantum correlations for bipartite states of two harmonic-oscillator subsystems, while for other values of the coefficients describing the environment, the asymptotic state is separable.

The results obtained can be a useful basis for describing the connection between uncertainty, decoherence, and correlations (entanglement) of open quantum systems with their environment. Due to the increasing interest manifested towards the continuous variables approach [23] to the theory of quantum information, these results, in particular, the possibility of maintaining a bipartite entanglement in a diffusive-dissipative environment even for asymptotic long times, could be useful for both phenomenological and experimental applications in the field of quantum information processing and communication.

\section{Acknowledgments}

The author acknowledges the financial support provided within the Project CEEX 68/2005.

\section{References}

1. E. Joos, H. D. Zeh, C. Kiefer, et al., Decoherence and the Appearance of a Classical World in Quantum Theory, Springer, Berlin (2003).

2. W. H. Zurek, Rev. Mod. Phys., 75, 715 (2003).

3. R. Alicki, Open Syst. Inform. Dyn., 11, 53 (2004).

4. M. A. Nielsen and I. L. Chuang, Quantum Computation and Quantum Information, Cambridge Univ. Press, Cambridge, MS (2000).

5. F. Benatti and R. Floreanini, Int. J. Mod. Phys. B, 19, 3063 (2005).

6. F. Benatti and R. Floreanini, J. Phys. A: Math. Gen., 39, 2689 (2006).

7. V. V. Dodonov, O. V. Man'ko, and V. I. Man'ko, J. Russ. Laser Res., 16, 1 (1995).

8. M. Morikawa, Phys. Rev. D, 42, 2929 (1990).

9. A. Peres, Phys. Rev. Lett., 77, 1413 (1996).

10. R. Simon, Phys. Rev. Lett., 84, 2726 (2000). 
11. G. Lindblad, Commun. Math. Phys., 48, 119 (1976).

12. A. Isar, A. Sandulescu, H. Scutaru, et al., Int. J. Mod. Phys. E, 3, 635 (1994).

13. V. V. Dodonov, E. V. Kurmyshev, and V. I. Man'ko, Phys. Lett. A, 79, 150 (1980).

14. S. S. Mizrahi, V. V. Dodonov, and D. Otero, Mod. Phys. Lett. B, 8, 995 (1994).

15. W. H. Zurek, S. Habib, and J. P. Paz, Phys. Rev. Lett., 70, 1187 (1993).

16. A. Isar, A. Sandulescu, and W. Scheid, Phys. Rev. E, 60, 6371 (1999).

17. V. V. Dodonov, S. S. Mizrahi, and A. L. de Souza Silva, J. Opt. B: Quantum Semiclass. Opt., 2, $271(2000)$.

18. A. Isar, Fortschr. Phys., 47, 855 (1999).

19. A. Isar and W. Scheid, Phys. Rev. A, 66, 042117 (2002).

20. A. Isar and W. Scheid, Physica A, 373, 298 (2007).

21. B. L. Hu and Y. Zhang, Int. J. Mod. Phys. A, 10, 4537 (1995).

22. A. Sandulescu, H. Scutaru, and W. Scheid, J. Phys. A: Math. Gen., 20, 2121 (1987).

23. S. L. Braunstein and A. K. Pati (eds.), Quantum Information with Continuous Variables, Kluwer, Dordrecht (2003). 